\title{
Comparison of SPIN and VIS for Protocol Verification
}

\author{
Hong Peng, Sofiène Tahar and Ferhat Khendek \\ Dept. of Electrical \& Computer Engineering, Concordia University \\ 1455 de Maisonneuve W., Montreal, Quebec, H3G 1M8 Canada \\ E-mail: \{pengh,tahar,khendek\}@ece.concordia.ca
}

The date of receipt and acceptance will be inserted by the editor

Key words: SPIN, VIS, Model Checking, Formal Ver-

ification, Protocols

\begin{abstract}
In this paper, we compare and contrast SPIN and VIS, two widely used formal verification tools. In particular, we devote a special attention to the efficiency of these tools for the verification of communications protocols that can be implemented either in software or hardware. As a basis of our comparison, we formally describe and verify the Asynchronous Transfer Mode Ring (ATMR) medium access protocol using SPIN and its hardware model using VIS. We believe that this study is of particular interest as more and more protocols, like ATM protocols, are implemented in hardware to match high speed requirements.
\end{abstract}

\section{Introduction}

For the last two decades, verification techniques have been applied successfully in software and hardware engineering. Various techniques have been proposed in the literature [6]. They range from pure simulation to model checking. The widely used simulation techniques cannot cover all design errors, especially for large systems. Like testing techniques, they are used to detect errors, but not to prove the correctness of the design. During the past decade, model checking techniques have established themselves as significant means for design validation, namely a given design is validated against specific and general properties. Two different fields of model checking have arisen: formal verification of software protocols and software systems, like SPIN [9], and formal verification of digital hardware, like VIS [2].

The SPIN software verification tool, developed by G. J. Holzmann at Bell Labs in 1989, is based on an interleaving model of concurrency, in which unlike hardware, 
only one component of the system state is allowed to change at a time. SPIN checks if the protocol specification is logically consistent. It reports errors in the protocol such as deadlock, livelock, or unreachable code. It also validates properties specified as linear time temporal logic (LTL) [8] formulas.

The VIS (Verification Interacting with Synthesis) tool, developed in 1995 by University of California at Berkeley and University of Colorado at Boulder, is based on synchronous models where any number of components can change state at a time. VIS integrates formal verification, simulation, and synthesis of finite-state hardware systems. It uses the Verilog hardware description language (HDL) as its input language. VIS supports branching time temporal logic (CTL) [8] symbolic model checking with fairness constrains [13].

The aim of this paper is to compare and contrast the SPIN (XSPIN version 3.3.3) and VIS (VIS release 1.3) tools using a software and a hardware model of the ATMR protocol [12] as a case study. We developed the software and hardware models independently and formally verified them in SPIN and VIS, respectively. Since the modeling language of SPIN and VIS are different, we cannot say explicitly that the two verified models, the VIS and the SPIN one, are exactly the same with respect to their semantics. However, we did follow the modeling and coding style of each of these tools. To expose the advantages and disadvantages of these two types of tools, we report and compare the verification CPU time, mem-

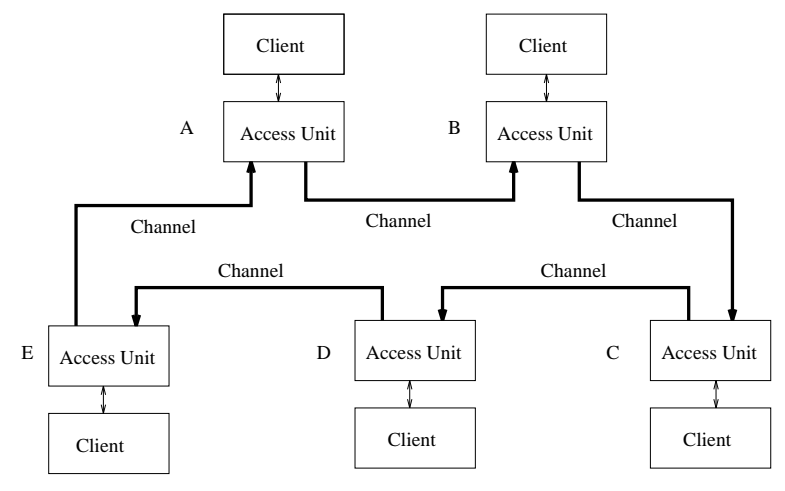

Fig. 1. ATMR structure with 5 nodes

ory usage, and state space generated. Furthermore, we describe the modeling techniques of asynchronous protocols in SPIN and VIS, and also analyze the source of the complexity in the verification.

The rest of the paper is structured as follows. We begin with an overview of the ATMR protocol (Section 2). We then describe the ATMR specification and verification in SPIN (Section 3) and VIS (Section 4), respectively. Finally, we conclude the paper with the comparison and contrast of SPIN and VIS (Section 5). The PROMELA and Verilog codes of the ATMR protocol are provided in the Appendix.

\section{ATM Ring Protocol}

The Asynchronous Transfer Mode Ring (ATMR) protocol [12] is an ISO standard based on a high speed shared medium connecting a number of access nodes by channels in a ring topology. Figure 1 gives an example ring with five nodes connected via a channel transferring cells between the nodes. For controlling access to this type of 


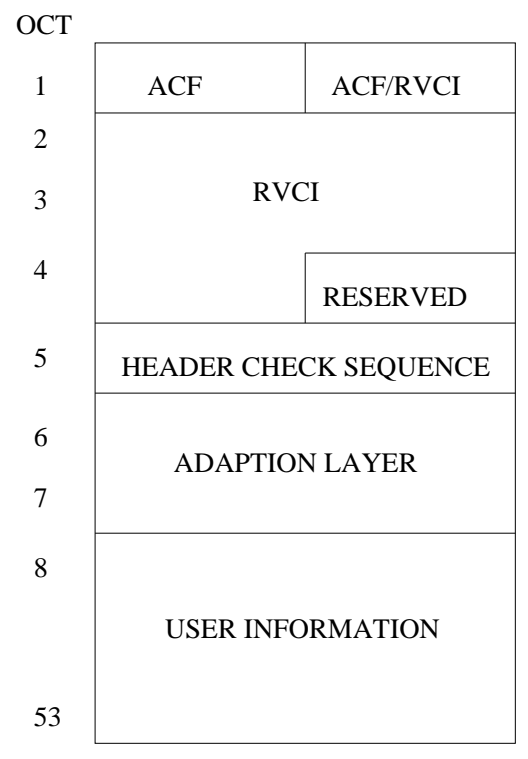

Fig. 2. Format of an ATMR cell

shared medium, the ring is first initialized with a fixed number of ATM cells continuously circulating around the channel from one node to another. Within each access node there is an access unit which performs both the physical layer convergence function and the access control function. Access to the ring is requested by the client and controlled by a combination of a window mechanism and a reset procedure. The client can issue a sending request to the access unit and receive a data cell. The window mechanism limits the number of cells a node can transmit at a time, called the "credits" of this node. The reset procedure reinitializes the window in all access units to a predefine credit value. The format of an ATMR cell is shown in Figure 2.

It contains an access control field (ACF), which includes a reset bit, a monitor bit and a busy address. When an access node releases an empty cell, it will fill

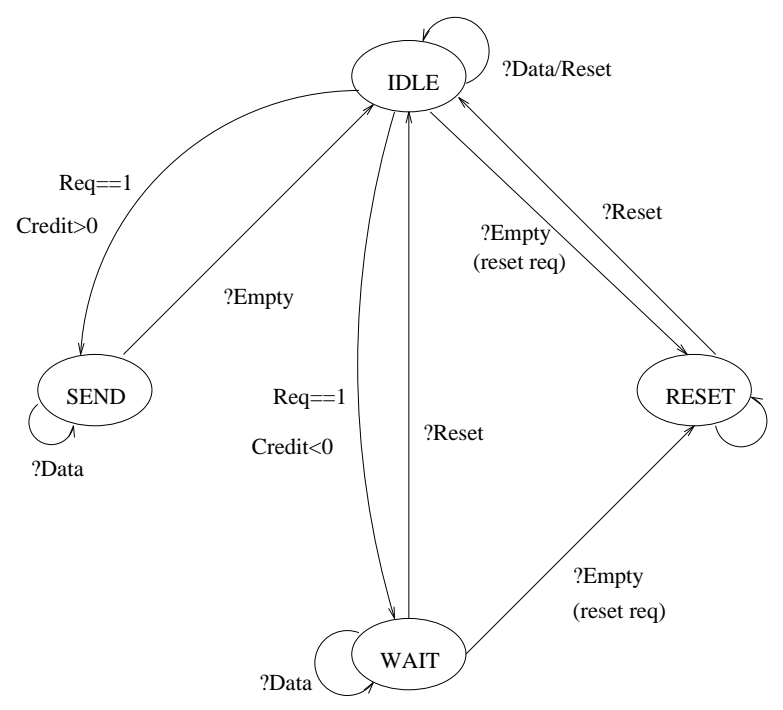

Fig. 3. FSM of an ATMR entity

its own address in the busy address field. The ATM cell is routed by using a ring virtual channel ID (RVCI) in the cell header.

The state transition diagram of the ATMR is shown in Figure 3, where "?" means receiving a message. The protocol entity of an access unit begins from an IDLE state. When the access unit has cells queued for transmission, it enters a SEND state and sends them in empty slots received at the ring interface with the address of the destination in the RVCI field of the cell header. The RVCI field in the header of all cells received at the ring interface of each node is checked and, if the cell is addressed to this node, the cell contents are copied and passed to the appropriate convergence sublayer. The RVCI field is then set to zero, which indicates an empty cell, and the cell is relayed to the next node in the ring. If a match is not found, then, this cell remains unchanged. 
Transmissions on the ring occur in cycles during which each access unit is allocated a fixed window size credit. This credit indicates the number of cells the access unit can transmit in this cycle before issuing or receiving a reset cell from the ring interface. A window credit counter is maintained by each access unit. Whenever this value is less than zero, the protocol entity enters a WAIT state to wait for a new credit. This value is initialized to the window size credit each time the ring is reset, namely the protocol entity is in a RESET state and the credit is decremented by one each time the access unit transmits a cell from its transmission queue. This mechanism is followed by all access units in the ring and hence eventually all units become inactive and the flow of cells around the ring ceases.

To reinitialize the transmission of the cells, an active access unit always overwrites its own address in the busy address field in the head of all cells passing the ring interface. This way, if an active access node receives a cell with its own address in the busy address field, it concludes that other nodes are inactive. Then after completely sending any remaining queued data from the higher layer, it creates a reset cell by setting the reset bit in the header of the next cell passing the ring interface. The reset cell circulates around the ring and causes all other access units to reinitialize their window credit counters. Once reinitialized, any access unit having data queued for transmission regains the active state and restarts sending cells.
The ATMR protocol was first modeled and checked by Charpentier and Padiou [4] who used UNITY to conduct a pencil-and-paper verification of it. Their validation abstracts away from any implementations, be it in software or in hardware. In next sections, we describe the modeling and verification of the ATMR protocol in SPIN and VIS, respectively.

\section{Verification Using SPIN}

SPIN [9] targets the verification of software systems and has been used in the past to trace design errors in distributed systems design, such as operating systems, data communications protocols, switching systems, concurrent algorithms, railway signaling protocols, etc. $[10,3]$. The tool checks the logical consistency of a protocol specification and reports design errors like deadlock, livelock, unreachable code and so on.

SPIN uses full LTL model checking, supporting all correctness requirements expressible in linear time temporal logic. It can also be used as an efficient on-thefly verifier for more basic safety and liveness properties. Many of the latter properties can be expressed, and verified, without the use of LTL though. Correctness properties can be specified as system or process invariants (using assertions), or as general linear temporal logic requirements (LTL), either directly in the syntax of nexttime free LTL, or indirectly as Büchi Automata (called 
never claims). If a property is invalid, an error trace is provided by the tool.

SPIN uses PROMELA (Process Meta Language) [9] as input modeling language. PROMELA allows abstractions in the protocol description by neglecting details that are irrelevant to process interaction. The intended use of SPIN is to verify fractions of process behavior, which for one reason or another are considered suspect. The relevant behavior is modeled in PROMELA and verified.

\subsection{ATMR Specification}

In order to test the capability of the SPIN tool, we tried to build a model as large as possible and let the tool do the reduction work. In this way, the verification engine works on its up-limit load, so that we can test the performance of the engine in a real situation. As an ATMR protocol can have $n$ nodes and $p$ channels [12], we will perform our verification on the model shown in Figure 1 including 3, 4, 5, and 6 ATMR nodes and a channel size of $6,8,10$, and 12 cells. The channel length between two neighboring nodes is two cells. We realized through experimentation that the five node model is the maximum model size that can make a comparison between SPIN and VIS within the memory available in the machine we used (Sun Sparc with 2 GB memory). However, for the purpose of comparison, we also put the experimental results of the ATMR model with three, four, five, and six nodes.
In the SPIN ATMR model, each node is specified as

a process

$$
\text { proctype Atmr(byte ID; chan in, out) }
$$

where $I D$ is the identification of the present node; in is the input channel and out is the output channel of the node. Since the nodes are in a ring form, the input channel of node $B$, for instance, will be the output channel of node $A$ (Figure 1 ).

Since SPIN's strength is in proving properties of interactions in a distributed system, but not in proving things about local computation or data dependency, we can try to make the model more general, more abstract. Namely, we will put only the behavior between the access unit and the channel into the model. Besides, we assume that the queue between the client and the access unit will be automatically refilled once it is empty. Thus, we can have a simple model while not affecting the behavior of the access unit.

An additional way of reducing the complexity is to remove everything that is not related to the property we are trying to prove, such as redundant data. For example, due to state space explosion, we did not succeed in verifying the whole data-path of the ATMR model. In order to simplify this latter, we abstract away all the information which will not affect the behavior of the ring accessing scheme, namely the HCS field, the adaption layer field and the user information field. The reduced cell format on which we based our verification is shown in Figure 4, where only 5 bits $\mathrm{ACF}$ and 3 bits RVCI 


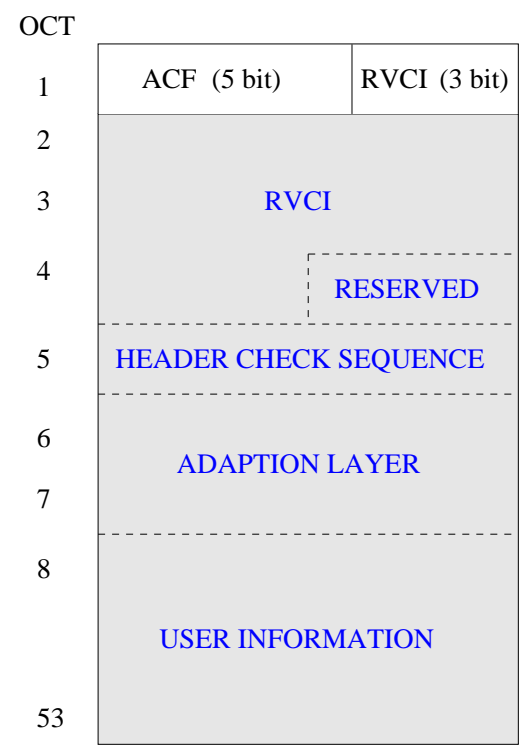

Fig. 4. Simplified Cell Format

will be used (non-shaded boxes in Figure 4). Because we kept all the access control information in the header format, namely the ACF and RVCI fields, the control behavior of ATMR with simplified cell format is exactly the same as that of the original one. After the reduction, the structure of the cell becomes:

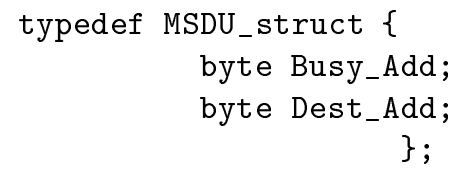

where Busy_Add is the busy address and Dest_Add is the destination address. MSDU_struct is the type definition of the cell. The cells are classified into DataCell, which contains user data, EmptyCell, which is available for loading, and ResetCell, which is to reset the credit of the access units in the ring.

Asynchronous channels are a significant source of complexity in the verification since there are lots of interleavings in the channel. Generally, the exclusive read/write option provided by SPIN is a good partial order reduction approach [11], which can reduce the verification CPU time.

Besides, in order to reduce the interleavings in the model, one of the possible solutions is to make as many statements as possible become atomic. For example, in the initialization process, we put all the initialization statements as atomic:

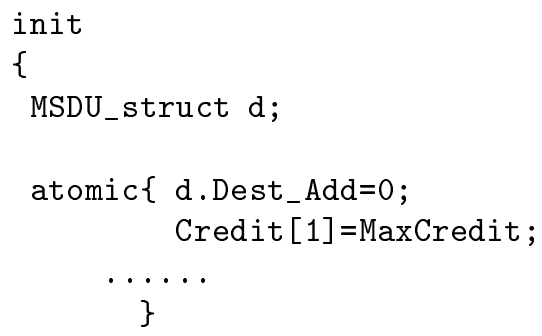

We can also reduce the interleavings of the model significantly by making atomic each state transition. For example, instead of

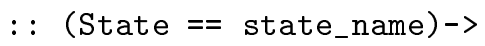

we can use

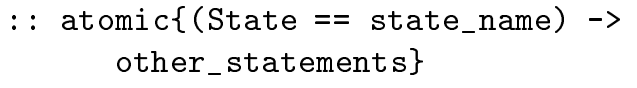

The exhaustive experiments we conducted show that the state space can be reduced for at least one order of magnitude in this way. However, in this case, the PROMELA model becomes synchronous which is not our intention. In the sequel, we did not use these atomic statements.

The PROMELA ATMR model is shown in Appendix A, where $I D$ is the identifier of this unit, and in and out are the incoming and outgoing channels of this unit, respectively. There are four states, Idle, Send, Reset, and 
WaitCredit. In each state, the unit can receive DataCell, EmptyCell, ResetCell.

An advantage of SPIN is that we can easily check deadlock using timeout statement in the model. Since in the deadlock status, the state transition stops, the timeout statement in a state can be easily checked out.

\subsection{ATMR Verification}

Once the ATMR model established, we validate it against a set of basic consistency properties. For illustration purposes, we present here six properties including liveness and safety. In the following descriptions, "[]", "<>", $"=="$ and " $\rightarrow$ " mean "always", "eventually", "logic equality" and "imply", respectively.

Property 1: Once an access unit exhausts its window size credit, the credit will eventually be renewed.

$$
[]((\text { credit }==0) \rightarrow<>(\text { credit }==6))
$$

where credit stands for the number of credits which is being held by an access unit and 6 is the preset maximum value.

Property 2: A client's request will be eventually acknowledged.

$$
[]((r e q==1) \rightarrow<>(a c k==1))
$$

where req is a cell sending request signal from a client to an access unit. If the requested cell has been sent out, the access unit will return an ack signal to the client.

Property 3: An access unit will eventually exit the RESET state and enter the SEND state.

$$
[]((\text { state }==R E S E T) \rightarrow<>(\text { state }==S E N D))
$$

where state stands for the current state of an access unit.

Property 4: An access unit will eventually exhaust its window size credit.

$$
[]((\text { credit }=6) \rightarrow<>(\text { credit }==0))
$$

here, 6 is the preset maximum credit value. We expect that all the credits will be consumed during the sending procedure.

Property 5: The number of reset cells in the ring cannot exceed the number of access units.

$$
[](N u m o f R s t<N u m o f U n i t)
$$

where NumofRst is the number of reset cells in the ring and NumofUnit is the number of access units.

Property 6: In the SEND state, a given station cannot send more cells than allowed by its credits.

$$
[]((\text { state }==S E N D) \rightarrow(\text { Outmsgs }<=6))
$$

here, Outmsgs is the number of cells sent by a given station in the SEND state.

The verification of the above six properties was performed on a Sun Sparc workstation with 2 GB of memory. We used two kinds of reachability analysis methods provided by the SPIN tool. One is the exhaustive exploration, the other is the supertrace/bitstate exploration which is an approximate approach, which can only provide maximum coverage search. In the ATMR verification, we first tried the exhaustive exploration. But this approach could not finish the ATMR verification due to an out of memory error, even when we applied the model compress techniques (-DCOLLAPSE, -DMA). 
Hong Peng, Sofiène Tahar and Ferhat Khendek: Comparison of SPIN and VIS for Protocol Verification

\begin{tabular}{|c|c|c|c|c|c|c|}
\hline \multirow[b]{2}{*}{ Property } & \multicolumn{3}{|c|}{3 nodes } & \multicolumn{3}{|c|}{4 nodes } \\
\hline & CPU Time (s) & Memory (MB) & States & CPU Time (s) & Memory (MB) & States \\
\hline $\mathrm{P} 1$ & 7.5 & 46.084 & 1335725 & 359.8 & 89.802 & $6.61796 \mathrm{e} 6$ \\
\hline $\mathrm{P} 2$ & 19.7 & 45.982 & 340879 & 941 & 87.345 & $1.56626 \mathrm{e} 7$ \\
\hline P3 & 5.2 & 46.084 & 122671 & 271.6 & 90.007 & $4.648 \mathrm{e} 6$ \\
\hline $\mathrm{P} 4$ & 18.6 & 46.084 & 390387 & 828.9 & 73.521 & $1.5796 \mathrm{e} 7$ \\
\hline $\mathrm{P} 5$ & 5.5 & 46.084 & 92322 & 236.6 & 89.086 & $4.3443 \mathrm{e} 6$ \\
\hline $\mathrm{P} 6$ & 3.2 & 44.982 & 101015 & 167.3 & 45.187 & $5.95003 \mathrm{e} 6$ \\
\hline \multirow[b]{2}{*}{ Property } & \multicolumn{3}{|c|}{5 nodes } & \multicolumn{3}{|c|}{6 nodes } \\
\hline & CPU Time (s) & Memory (MB) & States & CPU Time (s) & Memory (MB) & States \\
\hline $\mathrm{P} 1$ & 1632.5 & 127.225 & $3.26899 \mathrm{e} 7$ & 3261.4 & 1192.077 & $4.36862 \mathrm{e} 7$ \\
\hline $\mathrm{P} 2$ & 2273.6 & 264.906 & $3.41975 \mathrm{e} 7$ & 2883 & 264.19 & $4.98607 \mathrm{e} 7$ \\
\hline P3 & 2218.1 & 962.66 & $3.22636 \mathrm{e} 7$ & 2842.7 & 258.998 & $3.61413 \mathrm{e} 7$ \\
\hline $\mathrm{P} 4$ & 1255.3 & 258.25 & $2.9685 \mathrm{e} 7$ & 2783.9 & 298.487 & $3.69046 \mathrm{e} 7$ \\
\hline $\mathrm{P} 5$ & 2187.5 & 1441.086 & $4.32634 \mathrm{e} 7$ & - & - & - \\
\hline P6 & 1313.8 & 584.864 & $3.33867 \mathrm{e} 7$ & 2765 & 1729.955 & $4.53676 \mathrm{e} 7$ \\
\hline
\end{tabular}

Table 1. ATMR verification with SPIN

In contrast, the supertrace/bitstate (bit-state hashing) could finish the verification of the properties. Although the coverage is not one hundred percent, this latter still can give us some confidence about the correctness of the model. The supertrace/bitstate model checking experimental results are reported in Table 1 , including CPU time in seconds, memory usage in MB and the number of states stored. Graphic illustrations of the experimental results are plotted in Figures 5, 6, and 7.

From the graphic illustrations, we found that the increment of the state space is becoming steady when the model becomes larger, and so does the CPU time. This means that SPIN can handle larger models, while, affecting the state coverage (i.e., the number of visited stated relative to the number of actual states), however. Generally, For a hash-factor between 10 and 100, SPIN gives an expected coverage of $98 \%$ on average.

Bit-state hashing is an approximate approach. On the other hand, when compared with classical random simulation techniques, it is always better to use bit-state hashing because the coverage is usually much better than that achieved with random simulation. During the verification, we found that the more nodes are included in the ATMR model, the less is the coverage. In the 3-node verification, the coverage is greater than $99.9 \%$, but in the 6 -node verification, the coverage is less than $98 \%$.

There are some variance in the memory usage, especially in the 6-node model for Property 3 . We think there may be two reasons. One is that we are using the 


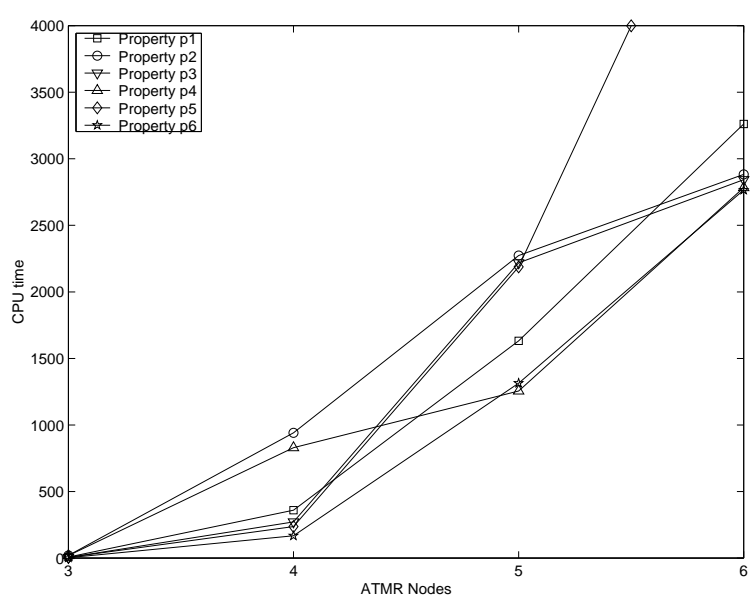

Fig. 5. SPIN verification CPU time

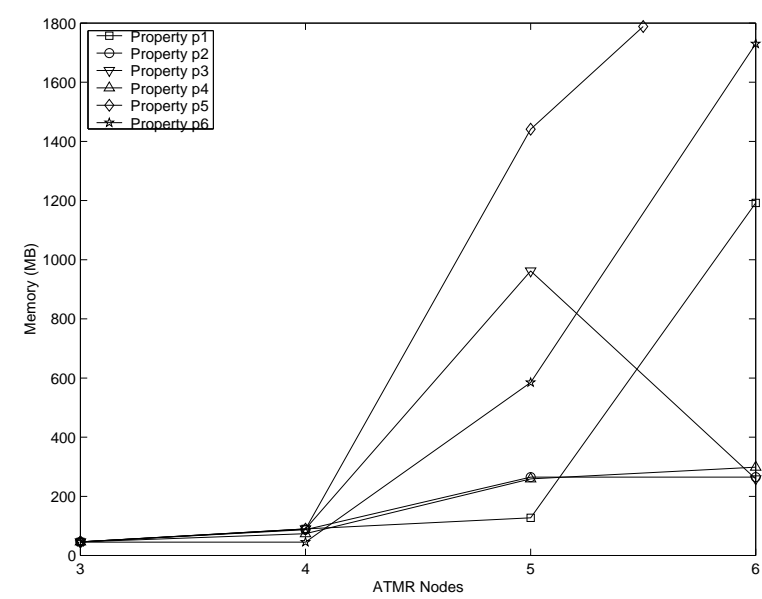

Fig. 6. SPIN verification memory usage

approximate method. This method is actually a "random" approach. The other is that we are working in a multi-user operating system. The variance in the system load will affect the experimental results.

\section{Verification Using VIS}

VIS [2] is a verification and synthesis tool for finite-state hardware systems, developed at University of California at Berkeley and University of Colorado at Boulder. It uses the Verilog HDL as the input language and supports

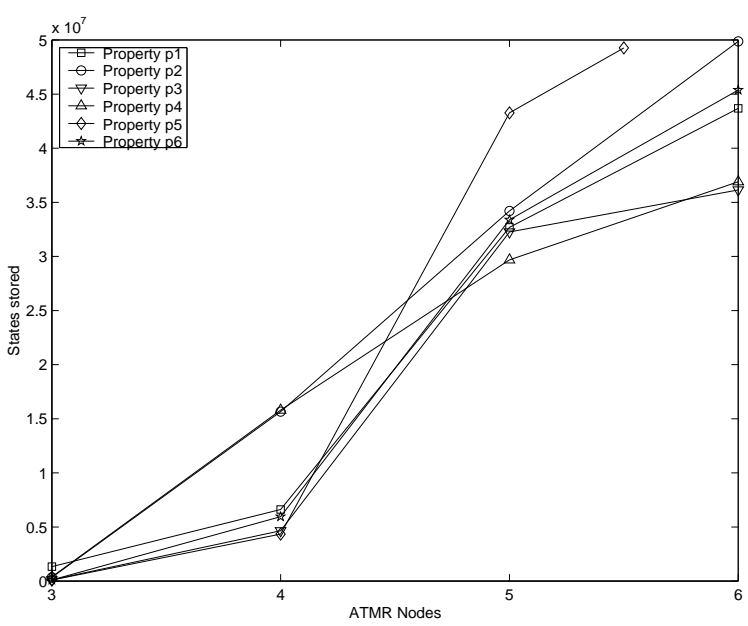

Fig. 7. SPIN verification state space

CTL model checking with fairness constrains. Its fundamental data structure is a multi-level network of latches and combinational gates. The variables of a network are multi-valued, and logic functions over these variables are represented by an extension of BDDs: multi-valued decision diagrams.

VIS operates on the intermediate format BLIF-MV [5]. It includes a compiler from Verilog to BLIF-MV and extracts a set of interacting FSMs that preserves the behavior of the Verilog program defined in terms of simulated results. Through the interacting FSMs, VIS performs CTL model checking under Büchi fairness constraints, i.e., sets of states that must be visited infinitely often. The language of a design is given by sequences over the set of reachable states that do not violate the fairness constraint. If model checking fails, VIS reports the failure with a counter-example. 


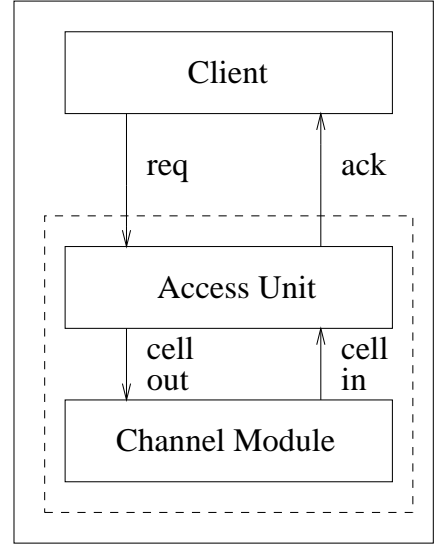

Fig. 8. Modified ATMR ring structure

Besides model checking, VIS supports equivalence checking, cycle-based simulation, and synthesis functions, such as state minimization and state encoding.

\subsection{ATMR Specification}

Since VIS is built on synchronous models, it is impossible to directly describe the original asynchronous ATMR in VIS, e.g., how to describe the cell transmission between two access units using synchronous Verilog. We hence need to build a pseudo-asynchronous ATMR protocol to simulate the ATMR protocol in the synchronous VIS environment. There are many methods to simulate an asynchronous system in a synchronous environment [1]. Here, because we only request that cell transmission be asynchronous and the module itself be synchronous, we propose to simply add a module channel in the Verilog specification. This channel model will play the role of a queue between two ATMR nodes (see Figure 8).
All the cells sent or received by the access unit will hence be queued in the channel module. When the access unit wants to read a cell from the channel, it actually reads the cell from the head of the queue. If the destination is the current node, the cell will be processed in this access unit. Otherwise, the cell will be forwarded to the next node via the channel module. This way, the sending and the receiving processes within the ring can remain asynchronous. The channel is defined as follows.

channel (ch_out, ch_in, ID);

where ch_out and ch_in are wired connections to and from the nodes; $I D$ is the identification of the channel. In this case, the access unit becomes.

mac_ctrl_node (req, ack, ch_out, ch_in, ID);

where req is the cell request signal from the client; ack is the acknowledgment; ch_out and ch_in are the output and input channels for each node; $I D$ is the identification of the node. Here, we do not put the clock signal because we use the implicit clock source provided by VIS. The req/ack pair follows the same rule as we defined in the SPIN modeling, namely once ack becomes true, req will be true in the next clock. Because Verilog instances are synchronized by the clock, we have to put the req generator in another instance and put a wire connection between these two instances.

Except above features, the ATMR model (Figure 9) we verified in VIS is very similar to that we used in SPIN. The cell format is here again a simplified one, containing only the ACF and RVCI fields (Figure 4). 


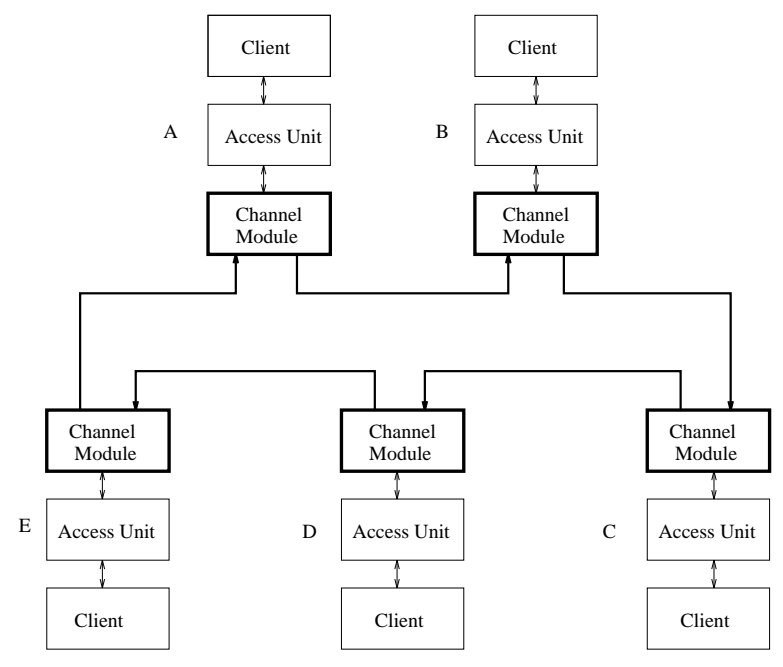

Fig. 9. Modified ATMR ring structure

Note that given the specification nature in SPIN and VIS, all components in VIS are true concurrent, while they are interleaved in SPIN.

The Verilog pseudo-asynchronous ATMR model is given in Appendix B, where $c l k$ is the system clock; req and ack are the signals from/to the clients; out_cell and in_cell are the output/input cells of this unit; id is the identifier of this unit. The states and the cell types are the same as that of SPIN model. The only difference is that because Verilog does not have chan (channel) data type and mtype (message type) variable, we have to examine the data bit in the cell format explicitly.

\subsection{ATMR Verification}

We verified the same properties as in the SPIN study. The only difference is that, in VIS, properties will be expressed in CTL and not in LTL. We present here the six liveness and safety properties of Section 3.2 in CTL. In the following descriptions, "=", " $\rightarrow$ " and "*" mean log- ical "equality", "implication" and "and", respectively.

"AG" and "AF" mean "all paths in all states" and "all paths in future states", respectively.

Property 1: Once an access unit exhausts its window size credits, the credits will eventually be renewed.

$$
\begin{aligned}
& A G(((\text { credit }[2]=0) *(\operatorname{credit}[1]=0) * \\
& (\operatorname{credit}[0]=0)) \rightarrow A F(\operatorname{credit}[2]=1) *(\operatorname{credit}[1]=1) * \\
& \text { credit }[0]=0))
\end{aligned}
$$

where credit is composed of three bits: credit [2], credit[1] and credit $[0]$.

Property 2: A client's request will eventually be acknowledged.

$$
A G((r e q=1) \rightarrow A F(a c k=1))
$$

Property 3: An access unit will eventually exit the RESET state and enter the SEND state (see Figure 4).

$$
A G((\text { state }=R E S E T) \rightarrow A F(\text { state }=S E N D)) ;
$$

Property 4: An access unit will eventually exhaust its window size credit.

$$
A G(((\operatorname{credit}[2]=1) *(\operatorname{credit}[1]=1) *
$$

$(\operatorname{credit}[0]=0)) \rightarrow A F((\operatorname{credit}[2]=0) *(\operatorname{credit}[1]=0) *$

$(\operatorname{credit}[0]=0)))$

In this property, we expect that all the credits will be consumed during the sending procedure.

Property 5: The number of reset cells in the ring cannot exceed the number of access units.

$$
A G(N u m o f R s t<N u m o f U n i t)
$$

In this property, NumofUnit is set to the number of access units in the verification, i.e., $3,4,5,6$, respectively. 
Property 6: At SEND state, a given station cannot send more cells than allowed by its credits.

$$
A G((\text { state }==S E N D) \rightarrow(\text { Outmsgs }<=6))
$$

here, Outmsgs is the number of cells which a given station sends at the SEND state.

The experimental results of the CTL model checking obtained in VIS are reported in Table 2, including CPU time in seconds, memory usage in $\mathrm{MB}$ and the number of BDD nodes allocated. The graphical representations are given in Figures 10, 11, and 12. These experiments were conducted on the same machine as the SPIN verification. During the verification, we used the advanced ordering, window and sift [2] to reduce the BDD/MDD size. VIS also provides a cone of influence model reduction [7] technique for invariant properties. However, in the verification of liveness properties, this technique cannot be applied. Besides, VIS provides a limited abstraction mechanism, namely the user must explicitly specify which signal in the model can be abstracted in one specific property verification. This technique, however, can only be used in a fairly simple situation and cannot be applied in our case. Since the modeling language of SPIN and VIS are different, we cannot say explicitly that the two verified models, the PROMELA one and the Verilog one, are exactly the same with respect to their semantics. However, what we did is trying to follow the modeling methods and coding styles of Verilog and PROMELA, respectively. We also tried to keep these two models to their minimum size in either tool, in order to be able to

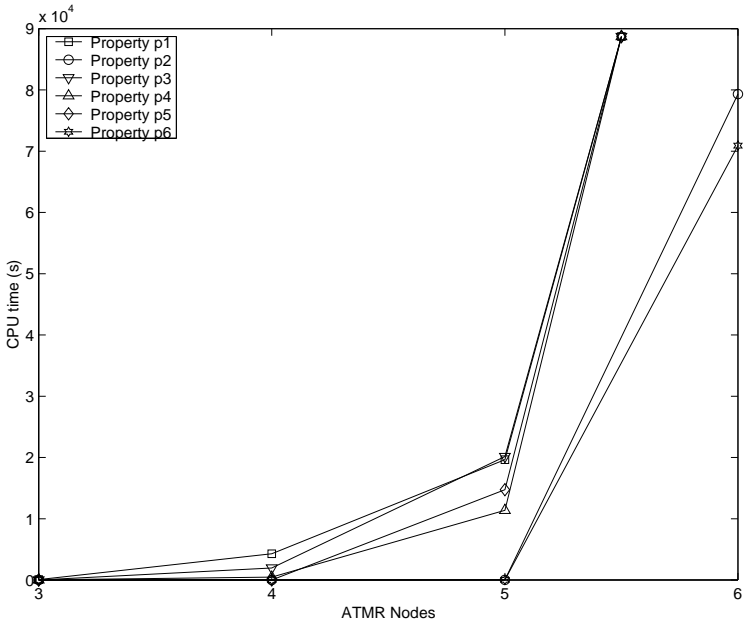

Fig. 10. VIS verification CPU time

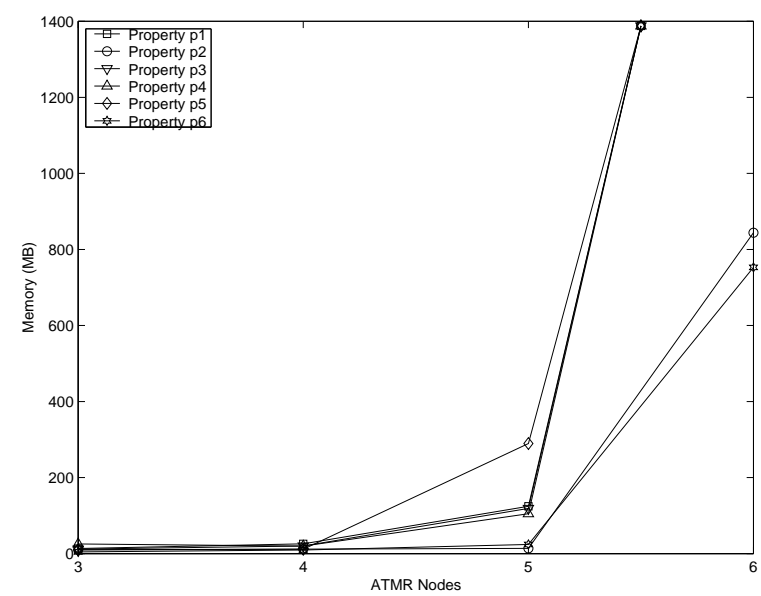

Fig. 11. VIS verification memory usage

compare the efficiency of SPIN and VIS in the verification of interleaving and concurrent models, respectively.

The VIS verification approach is not directly scalable to large designs due to state space explosion. From the verification results, we see that in the $3,4,5$-node model, the verification can be finished. However, the state space blows up quickly with respect to the model size. In the verification of the 6-node model, only Property 2 can be finished. The other properties fail short of memory. 
Hong Peng, Sofiène Tahar and Ferhat Khendek: Comparison of SPIN and VIS for Protocol Verification

\begin{tabular}{|c|c|c|c|c|c|c|}
\hline \multirow[b]{2}{*}{ Property } & \multicolumn{3}{|c|}{3 nodes } & \multicolumn{3}{|c|}{4 nodes } \\
\hline & CPU Time (s) & Memory (MB) & States & CPU Time (s) & Memory (MB) & States \\
\hline $\mathrm{P} 1$ & 57 & 13.59 & 1513196 & 4290 & 26 & 680963412 \\
\hline $\mathrm{P} 2$ & 4.6 & 10.04 & 318106 & 10.1 & 11.85 & 732167 \\
\hline P3 & 6.3 & 11.91 & 528411 & 1962.1 & 201.69 & 346168207 \\
\hline $\mathrm{P} 4$ & 25.6 & 13.72 & 1166100 & 467.7 & 19.99 & 83736894 \\
\hline P5 & 4.1 & 9.87 & 308435 & 8.1 & 11.72 & 550583 \\
\hline P6 & 4.8 & 9.98 & 314168 & 9.6 & 11.66 & 565248 \\
\hline \multirow[b]{2}{*}{ Property } & \multicolumn{3}{|c|}{5 nodes } & \multicolumn{3}{|c|}{6 nodes } \\
\hline & CPU Time (s) & Memory (MB) & States & CPU Time (s) & Memory (MB) & States \\
\hline $\mathrm{P} 1$ & 19640.7 & 124.49 & 1811363276 & - & - & - \\
\hline $\mathrm{P} 2$ & 31 & 13.79 & 3920178 & 79324.5 & 844.01 & 3947015525 \\
\hline P3 & 20146.9 & 118.98 & 1829837953 & - & - & - \\
\hline $\mathrm{P} 4$ & 11372.3 & 105.04 & 1152672565 & - & - & - \\
\hline P5 & 14734.3 & 289.57 & 1231510174 & - & - & - \\
\hline P6 & 24.2 & 13.77 & 2492626 & 70920.1 & 752.69 & 2606471216 \\
\hline
\end{tabular}

Table 2. ATMR verification with VIS

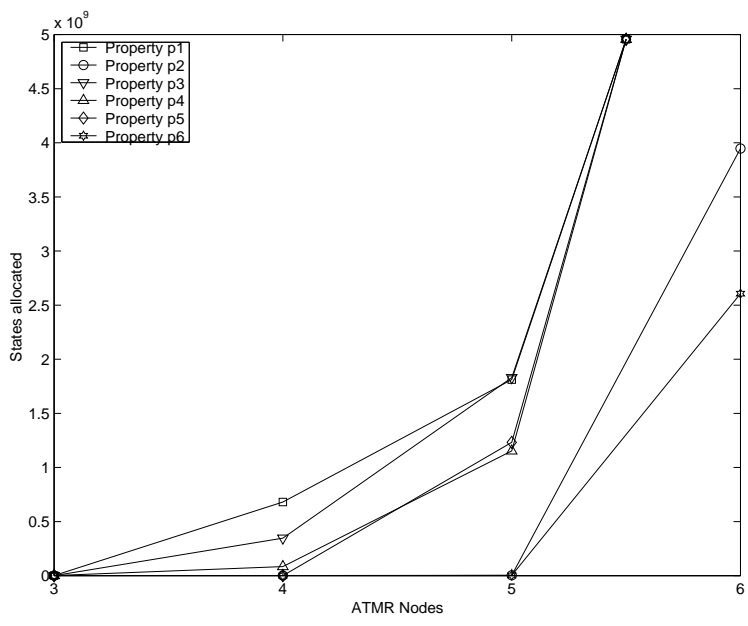

Fig. 12. VIS verification state space

There are two reasons for the state space explosion. One is the introduction of the channel module which is composed of 19 latches. The other is the circular dependency of the nodes in the ring. To solve this problem, we believe that the data complexity must be decreased by more efficient abstraction and reduction techniques. Finally, for small models (less than 6 node), we found out that the memory usage in VIS is more efficient than SPIN since VIS can finish an exhaustive search.

\section{Conclusions}

In this paper, we formally verified the asynchronous ATMR protocol in both SPIN and VIS. Generally, when a protocol is implemented in hardware, it cannot be handled only by a software (protocol) verification tool, like SPIN, since most of these tools are based on an interleaving current model and cannot reflect the true concurrency aspects of a hardware implementation. A verification in VIS leaves us, however, with the obligation of simulating an asynchronous protocol in a synchronous environment. 


\begin{tabular}{|c||c|c|}
\hline Feature & SPIN & VIS \\
\hline \hline Target system & Software & Hardware \\
\hline Basic model & Interleaving model & Synchronous model \\
\hline Property language & LTL & CTL \\
\hline Specification language & PROMELA & Slower \\
\hline Verif. of asynch. protocol & Yes & Additional channel module \\
\hline CPU time usage & Faster & Smaller \\
\hline Main memory usage & Larger & No \\
\hline Detect dead-lock, live-lock, etc. & Yes & Indirectly via temporal formulas \\
\hline Graphic User Interface & Yes & PIS \\
\hline
\end{tabular}

Table 3. Comparison of SPIN and VIS

Because of the inherent weakness of model checking, both SPIN and VIS are not directly scalable to large designs due to state space explosion. Thus, it is important to find techniques that can be used in conjunction with model checking tools like SPIN and VIS to extend the size of the systems that can be verified. In this paper, we used a data abstraction approach to reduce the model of the ATMR protocol for both the SPIN and VIS verifications.

Unlike VIS, SPIN is based on interleaving models, and hence runs generally faster than VIS because each state update is a simpler operation, being restricted to one component only. Comparing the two sets of verification results, we can find generally the verification in SPIN is faster. For example, in the 3-node model verification, the verifications of Properties $1,3,4$ and 6 in SPIN are faster than those in VIS. Although the approximate technique used in the SPIN verification may contribute to this difference, we do not think this is the major factor because the SPIN coverages of the 3-node model properties are greater than 99.9 percent. From this point of view, it is a disadvantage for VIS not providing an easy-to-use approximate technique. In SPIN, one possible way to reduce the interleavings is to make the statements atomic if these statements can be synchronous. Experiments showed that in this way the state space can be reduced for at least one order of magnitude.

SPIN uses explicit state enumeration while VIS uses implicit state enumeration (symbolic model checking). Generally, VIS can use the memory more efficiently. From our experiments, we found that VIS can finish the exhaustive search in the 3,4 , and 5 -node models. The onthe-fly approach in SPIN does not show advantages because the model is large and the properties are global.

Since both SPIN and VIS are not scalable to large designs, model reduction techniques are very important for verification. Both tools provide model reduction options, namely partial order and cone of influence, respectively. Partial order reduction can only be used in the interleaving model and is not feasible in a tool like VIS. The 
model reduction techniques in VIS are limited and need a lot of human interaction.

Another weakness in VIS is that it cannot directly report deadlocks, livelock and unreachable code. We have to express these properties with temporal formulas. For example, a deadlock, is expressed as: "Sender is not in send state and receiver is not in receiving state and there is at least one cell in the channel." Generally, this property is difficult to specify in CTL. In SPIN, a deadlock can be easily found using a timeout statement.

Finally, two practical features of these tools are worth mentioning. Namely, while VIS has a Verilog front-end allowing industrial designs to be imported and verified, SPIN comes with a graphic user's interface which greatly eases the use of the tool compared to VIS.

A summary of the main comparison mentioned above and throughout the paper is given in Table 3 .

\section{Acknowledgments}

This work is partially supported by a Concordia graduate student scholarship and NSERC research grants no.

OGP0194302 and no. OGP0194234.

\section{References}

1. Rajeev Alur and Thomas A. Henzinger. Reactive modules. Formal Methods in System Design: An International Journal, 15(1):7-48, July 1999.

2. R. K. Brayton et al. VIS: A system for verification and synthesis. In Proceedings of Computer Aided Verification, volume 1102 of $L N C S$, pages 428-432. Springer Verlag, Rutgers University, NY, USA, July 1996.
3. E. Brinksma and A. Mader. Verification and optimization of a PLC control schedule. In Proceedings of the rth SPIN Workshop, pages 73-92, Stanford University, California, USA, September 2000.

4. M. Charpentier and G. Padiou. Specification and verification of the ATMR protocol using UNITY. In Proceedings of International Workshop on Formal Methods for Parallel Programming, pages 26-36, University of Geneva, Switzerland, April 1997.

5. S. T. Cheng, R. K. Brayton, G. York, K. A. Yelick, and A. Saldanha. Compiling verilog into timed finite state machines. In Proceedings of International Verilog Conference, 1995.

6. E. M. Clarke, O. Grumberg, and D. Long. Verification tools for finite-state concurrent systems. In $R E X$ School/symposium on a Decade of Concurrency: Reflections and Perspectives, pages 124-175, Noordwijkerhout, The Netherlands, June 1993.

7. E. M. Clarke, O. Grumberg, and D. Peled. Model Checking. MIT Press, 2000.

8. E. A. Emerson. Temporal and modal logic, Handbook of theoretical computer science. Elsevier Sciences B.V. J. van leeuwn north Holland edition, 1990.

9. G. J. Holzmann. Design and validation of computer protocols. Prentice Hall, 1991.

10. G. J. Holzmann. The engineering of a model checker: the Gnu i-protocol case study revisited. In Proceedings of Spin Workshop, pages 233-244, Toulouse, France, September 1999.

11. G.J. Holzmann and D. Peled. An improvement in formal verification. In Proceedings of International Conference on Formal Description Techniques for Distributed Systems and Communications Protocols, pages 177-194, Bern, Switzerland, September 1994.

12. ISO. Specification of the Asynchronous Transfer Mode Ring (ATMR) Protocol, 2.0 edition, January 1993.

13. K. L. McMillan. Symbolic Model Checking. Kluwer Academic Publishers, 1993. 


\section{A PROMELA model of the ATMR}

proctype AccessUnit (byte ID; chan in, out) \{ byte State=Idle;

MSDU_struct data;

$\mathrm{xr}$ in;

xs out;

start: do

$::($ State $==$ Idle $)->$

if

$::($ Msgs $[\mathrm{ID}]>0)->$

if

: : (Credit $[$ ID $]>0)->$ State $=$ Send ;

goto start;

: : (Credit $[$ ID] <=0) $->$

State=WaitCredit; goto start;

fi

$::($ Msgs $[I D]==0)->$

Msgs [ID] =MaxMsgs ;

$\operatorname{ack}[\mathrm{ID}]=0 ; \operatorname{req}[\mathrm{ID}]=1$;

if

: : in?DataCell (data)->

if

$::($ data.Dest_Add==ID) $->$

data. Dest_Add=0;

out ! EmptyCell (data);

: : (data.Dest_Add!=ID) -> out! DataCell (data);

$f i$

: : in?ResetCell (data)->

Credit [ID] =MaxCredit;

out ! ResetCell (data);

: : in?EmptyCell (data)->

if

: : (data.Busy_Add==ID) $->$

Receive_RstReq[ID]=1;

out ! ResetCell (data) ;

Send_Rst $[I D]=1$;

State=Reset ;

$::($ data.Busy_Add $!=I D)->$

out ! EmptyCell (data);

State=Idle;

fi;

Msgs $[I D]=$

( (A [ID $) *$ PreMsgs $[$ ID $]+C[I D])$;

$\operatorname{ack}[I D]=0 ; \operatorname{req}[I D]=1$;

PreMsgs [ID] =Msgs [ID] ;

fi;

fi;

$::($ State $==$ Send $)->$

if

: : in?EmptyCell (data)->

data.Dest_Add=

( (A [ID] *PreDest [ID] +C [ID] ) ;

if

: : (data.Dest_Add==ID) ->

data.Dest_Add= (data. Dest_Add;
$::$ (data.Dest_Add $!=I D)->$;

$\mathrm{f} i$;

PreDest $[$ ID $]=$ data. Dest_Add;

data.Busy_Add=ID;

out! DataCell (data);

Msgs [ID]-- ;

$\operatorname{ack}[I D]=1 ; \operatorname{req}[I D]=0$;

Credit [ID]--; State=Idle;

: : in?DataCell (data)->

data.Busy_Add=ID;

if

$:$ : (data. Dest_Add==ID) ->

data.Busy_Add=ID;

data.Dest_Add=0;

out ! EmptyCell (data) ;

$:$ : (data.Dest_Add !=ID) ->

data.Busy_Add=ID;

out! DataCell (data);

fi

: : in?ResetCell (data)->

Credit [ID] =MaxCredit ;

out ! ResetCell (data) ;

State=Idle;

fi

$::($ State==Reset $)->$

if

: : in?DataCell (data)->

if

$::$ (data.Dest_Add==ID) $->$

data. Dest_Add=0;

out !EmptyCell (data);

: : (data.Dest_Add!=ID) ->

out! DataCell (data);

fi

: : in?ResetCell (data)->

Credit [ID] =MaxCredit;

Send_Rst $[I D]=0$;

Receive_RstReq[ID] $=0$;

data.Busy_Add=ID;

out ! EmptyCell (data) ;

State=Idle;

: : in?EmptyCell (data)->

out ! EmptyCell (data) ;

: :timeout->

data.Busy_Add=ID

out! ResetCell (data) ; NumofRst++;

$\mathrm{fi}$

$::($ State==WaitCredit) $->$

if

: : in?DataCell(data)->

if

$:$ : (data.Dest_Add==ID) $->$

data.Dest_Add=0;

out !EmptyCell (data);

: : (data.Dest_Add! =ID) ->

out! DataCell (data);

fi 


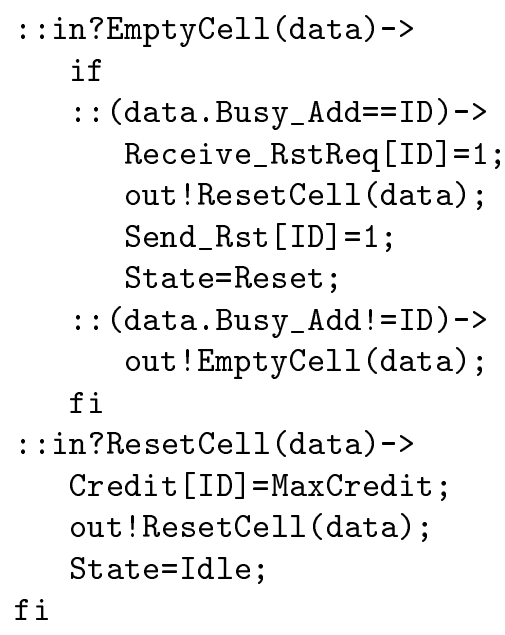

\section{B Verilog model of the ATMR}

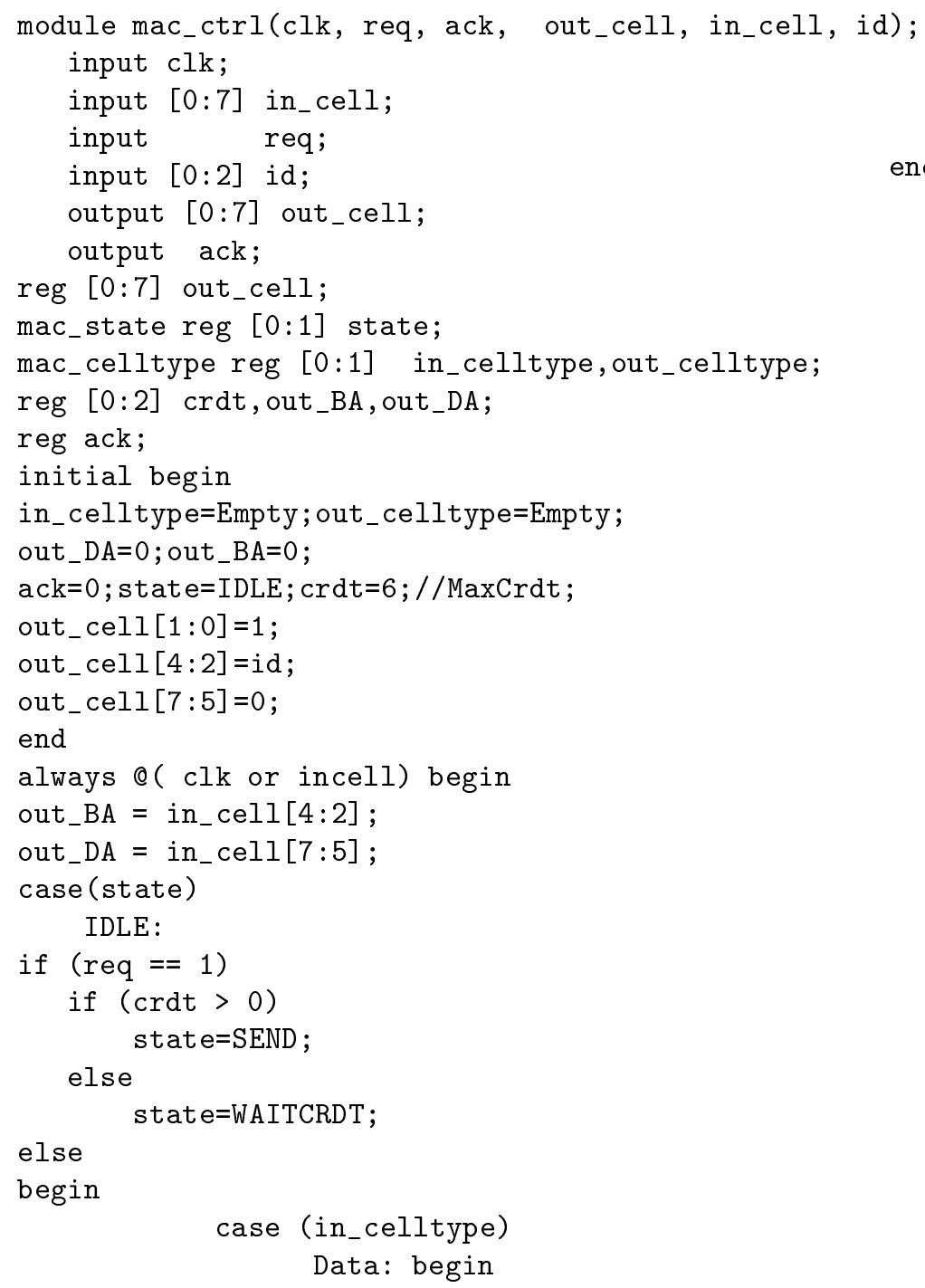

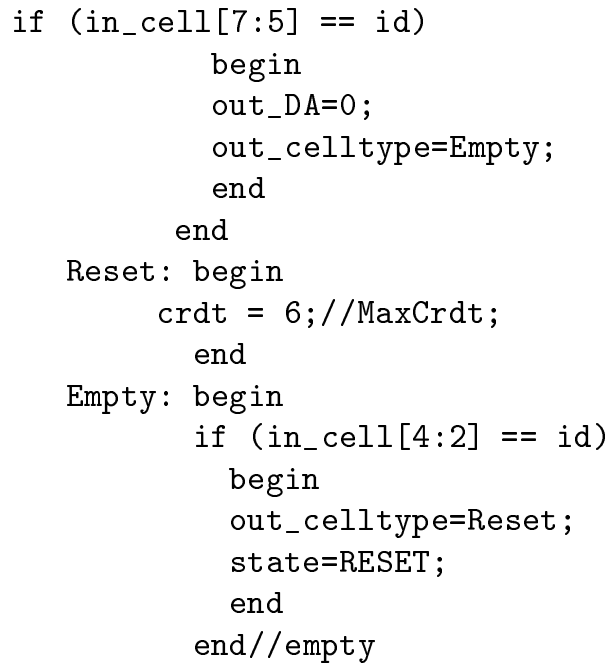

end

SEND :

case (in_celltype) Empty: begin out_celltype=Data ; out_BA=id ;

end

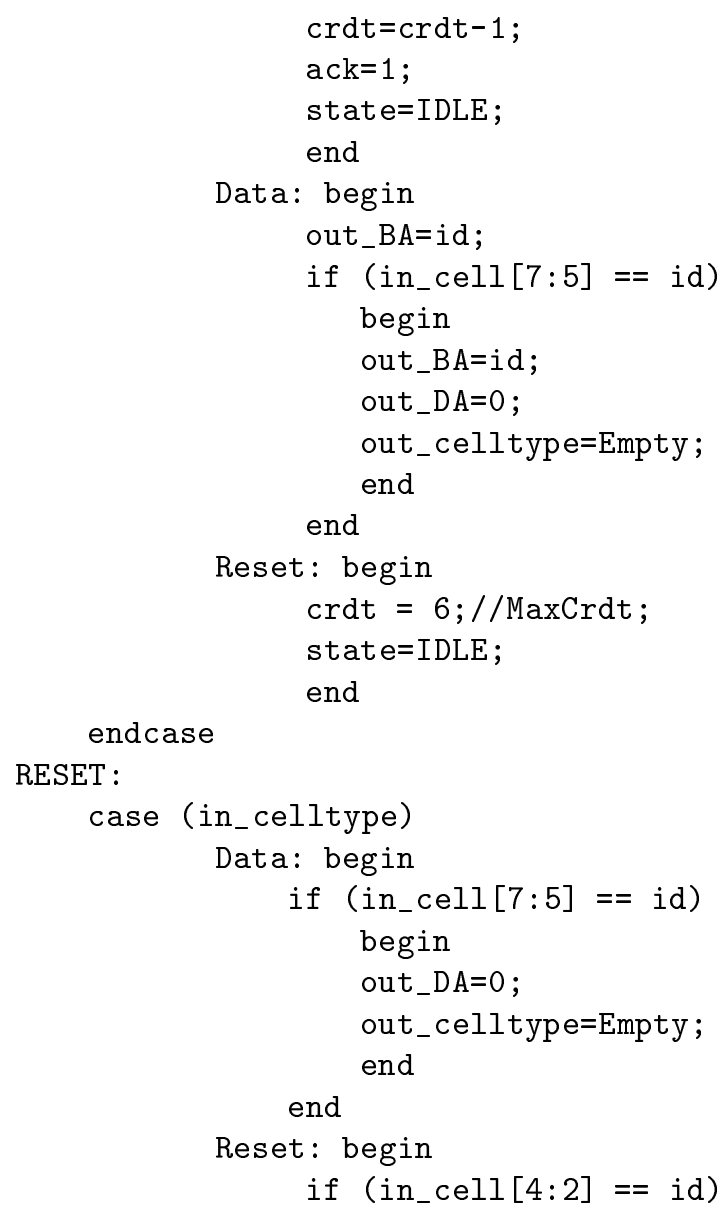




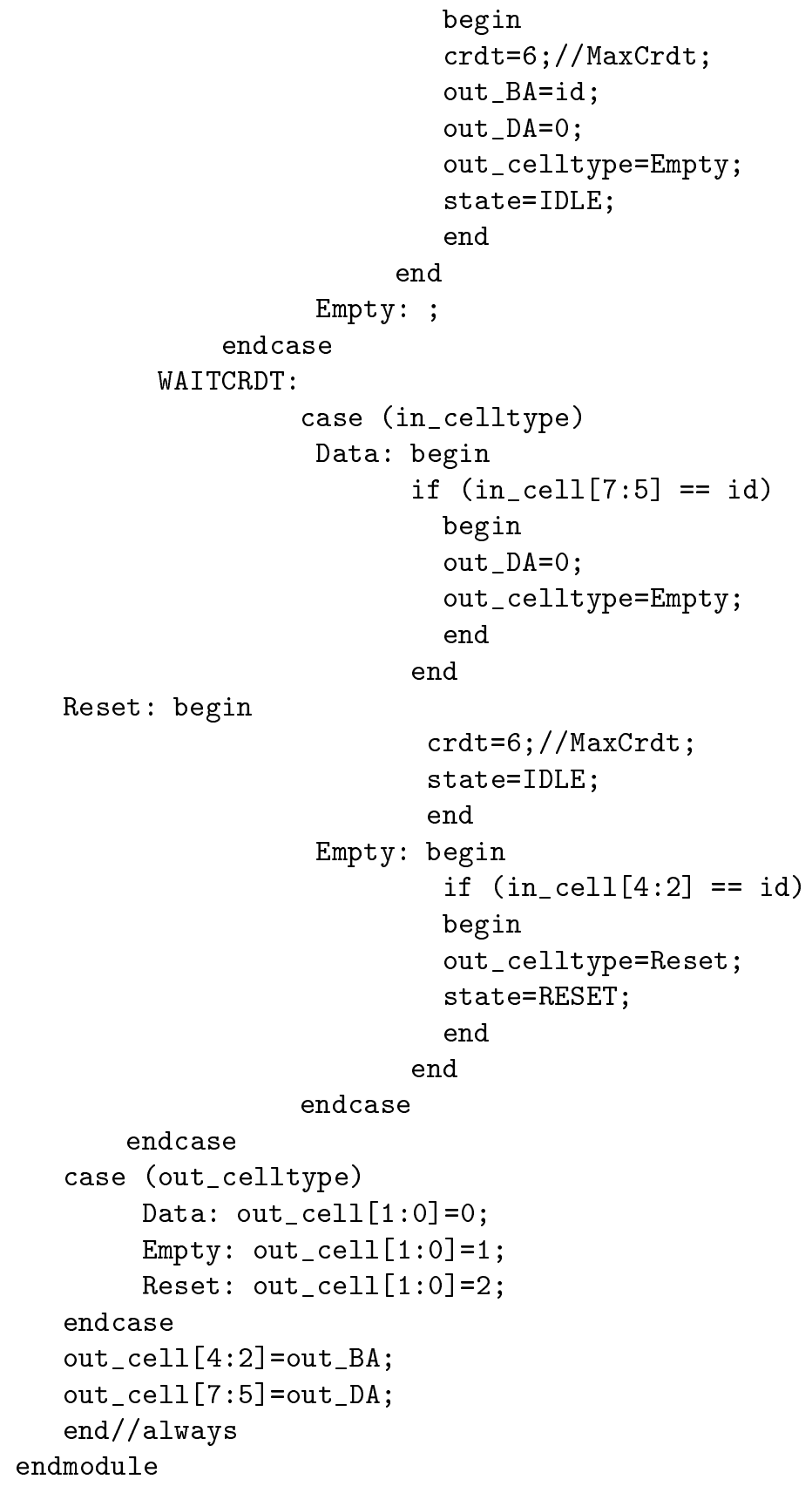

